\title{
Existence and Uniqueness of Solution for a Class of Nonlinear Degenerate Elliptic Equations
}

Albo Carlos Cavalheiro*

Department of Mathematics, State University of Londrina, Londrina, Brazil

Received 21 November 2018; Accepted (in revised version) 29 September 2019

Abstract. In this work we are interested in the existence and uniqueness of solutions for the Navier problem associated to the degenerate nonlinear elliptic equations

$$
\begin{aligned}
& \Delta\left[\omega_{1}(x)|\Delta u|^{p-2} \Delta u+v_{1}(x)|\Delta u|^{q-2} \Delta u\right] \\
& \quad-\operatorname{div}\left[\omega_{2}(x)|\nabla u|^{r-2} \nabla u+v_{2}(x)|\nabla u|^{s-2} \nabla u\right] \\
= & f(x)-\operatorname{div}(G(x)) \text { in } \Omega,
\end{aligned}
$$

in the setting of the weighted Sobolev spaces.

Key Words: Degenerate nonlinear elliptic equation, Weighted Sobolev spaces.

AMS Subject Classifications: 35J60, 35J70

\section{Introduction}

In this work we prove the existence and uniqueness of (weak) solutions in the weighted Sobolev space $X=W^{2, p}\left(\Omega, \omega_{1}\right) \cap W_{0}^{1, r}\left(\Omega, \omega_{2}\right)$ (see Definition 2.4 and Definition 2.5 for the Navier problem

$$
\text { (P) } \begin{cases}L u(x)=f(x)-\operatorname{div}(G(x)) & \text { in } \Omega, \\ u(x)=\Delta u(x)=0 & \text { on } \partial \Omega,\end{cases}
$$

where $L$ is the partial differential operator

$$
\begin{aligned}
L u(x)=\Delta & {\left[\omega_{1}(x)|\Delta u|^{p-2} \Delta u+v_{1}(x)|\Delta u|^{q-2} \Delta u\right] } \\
& -\operatorname{div}\left[\omega_{2}(x)|\nabla u|^{r-2} \nabla u+v_{2}(x)|\nabla u|^{s-2} \nabla u\right],
\end{aligned}
$$

*Corresponding author. Email address: accava@gmail .com (A. C. Cavalheiro) 
where $\Omega$ is a bounded open set in $\mathbb{R}^{n}, \omega_{1}, \omega_{2}, v_{1}$ and $v_{2}$ are four weight functions, $\Delta$ is the Laplacian operator and $2 \leq q, s<r<p<\infty$.

Let $\Omega$ be an open set in $\mathbb{R}^{n}$. We denote by $\mathcal{W}(\Omega)$ the set of all measurable, a.e. in $\Omega$ positive and finite functions $\omega=\omega(x), x \in \Omega$. Elements of $\mathcal{W}(\Omega)$ will be called weight functions. Every weight $\omega$ gives rise to a measure on the measurable subsets of $\mathbb{R}^{n}$ through integration. This measure will be denoted by $\mu_{\omega}$. Thus,

$$
\mu_{\omega}(E)=\int_{E} \omega(x) d x
$$

for measurable sets $E \subset \mathbb{R}^{n}$.

In general, the Sobolev spaces $\mathrm{W}^{k, p}(\Omega)$ without weights occur as spaces of solutions for elliptic and parabolic partial differential equations. For degenerate partial differential equations, i.e., equations with various types of singularities in the coefficients, it is natural to look for solutions in weighted Sobolev spaces (see [1-3,5,10] and [15]).

A class of weights, which is particularly well understood, is the class of $A_{p}$-weights (or Muckenhoupt class) that was introduced by B. Muckenhoupt (see [12]). These classes have found many useful applications in harmonic analysis (see [14]). Another reason for studying $A_{p}$-weights is the fact that powers of the distance to submanifolds of $\mathbb{R}^{n}$ often belong to $A_{p}$ (see [11]). There are, in fact, many interesting examples of weights (see [10] for $p$-admissible weights).

In the non-degenerate case (i.e., with $\omega(x) \equiv 1$ ), for all $f \in L^{p}(\Omega)$ the Poisson equation associated with the Dirichlet problem

$$
\begin{cases}-\Delta u=f(x) & \text { in } \Omega, \\ u(x)=0 & \text { on } \partial \Omega,\end{cases}
$$

is uniquely solvable in $W^{2, p}(\Omega) \cap W_{0}^{1, p}(\Omega)$ (see [9]), and the nonlinear Dirichlet problem

$$
\begin{cases}-\Delta_{p} u=f(x) & \text { in } \Omega, \\ u(x)=0 & \text { on } \partial \Omega,\end{cases}
$$

is uniquely solvable in $W_{0}^{1, p}(\Omega)$ (see [3]), where $\Delta_{p} u=\operatorname{div}\left(|\nabla u|^{p-2} \nabla u\right)$ is the $p$-Laplacian operator. In the degenerate case, the weighted $p$-Biharmonic operator has been studied by many authors (see [13] and the references therein), and the degenerated $p$-Laplacian has been studied in [5]. The problem with degenerated $p$-Laplacian and $p$-Biharmonic operators in the case $\omega_{1}=\omega_{2}=v_{1}=v_{2}$ and $p=q=r=s$

$$
\begin{cases}\Delta\left(\omega(x)|\Delta u|^{p-2} \Delta u\right)-\operatorname{div}\left[\omega(x)|\nabla u|^{p-2} \nabla u\right]=f(x)-\operatorname{div}(G(x)) & \text { in } \Omega, \\ u(x)=\Delta u(x)=0 & \text { on } \partial \Omega,\end{cases}
$$

has been studied by the author in [2].

The following theorem will be proved in Section 3 . 
Theorem 1.1. Let $2 \leq q, s<r<p<\infty$ and $\Omega \subset \mathbb{R}^{n}$ is a bounded open set. Assume

(H1) $\omega_{1} \in A_{p}, \omega_{2} \in A_{r}$ and $v_{1}, v_{2} \in \mathcal{W}(\Omega)$;

(H2) $\frac{v_{1}}{\omega_{1}} \in L^{p /(p-q)}\left(\Omega, \omega_{1}\right)$ and $\frac{v_{2}}{\omega_{2}} \in L^{r /(r-s)}\left(\Omega, \omega_{2}\right)$;

(H3) $\frac{f}{\omega_{2}} \in L^{r^{\prime}}\left(\Omega, \omega_{2}\right)$ and $\frac{|G|}{\omega_{2}} \in L^{r^{\prime}}\left(\Omega, \omega_{2}\right)$, where $G=\left(g_{1}, \cdots, g_{n}\right)$.

Then the problem $(P)$ has a unique solution $u \in X=W^{2, p}\left(\Omega, \omega_{1}\right) \cap W_{0}^{1, r}\left(\Omega, \omega_{2}\right)$. Moreover, since $0<\frac{1}{p}+\frac{1}{r}<1$, then

$$
\|u\|_{X} \leq C_{p, r}\left(\frac{M^{p^{\prime}-1}}{p^{\prime}}+\frac{M^{r^{\prime}-1}}{r^{\prime}}\right)
$$

where

$$
M=C_{\Omega}\left\|f / \omega_{2}\right\|_{L^{r^{\prime}}\left(\Omega, \omega_{2}\right)}+\left\||G| / \omega_{2}\right\|_{L^{r^{\prime}}\left(\Omega, \omega_{2}\right)},
$$

$C_{\Omega}$ is the constant in Theorem 2.2 and $C_{p, r}=p r /(p r-p-r)$.

\section{Definitions and basic results}

Definition 2.1. Let $1 \leq p<\infty$. A weight $\omega$ is said to be an $A_{p}$-weight, if there is a positive constant $C=C(p, \omega)$ such that, for every ball $B \subset \mathbb{R}^{n}$

$$
\begin{array}{ll}
\left(\frac{1}{|B|} \int_{B} \omega(x) d x\right)\left(\frac{1}{|B|} \int_{B} \omega^{1 /(1-p)}(x) d x\right)^{p-1} \leq C, & \text { if } p>1, \\
\left(\frac{1}{|B|} \int_{B} \omega(x) d x\right)\left(\operatorname{ess} \sup _{x \in B} \frac{1}{\omega(x)}\right) \leq C, & \text { if } p=1,
\end{array}
$$

where $|\cdot|$ denotes the $n$-dimensional Lebesgue measure in $\mathbb{R}^{n}$.

If $1<q \leq p$, then $A_{q} \subset A_{p}$ (see [8,10] or [14] for more information about $A_{p}$-weights). We say the weight $\omega$ satisfies the doubling condition if there exists a positive constant $C$ such that $\mu(B(x ; 2 r)) \leq C \mu(B(x ; r))$ for every ball $B=B(x ; r) \subset \mathbb{R}^{n}$, where $\mu(B)=$ $\int_{B} \omega(x) d x$. If $\omega \in A_{p}$, then $\mu$ is doubling (see Corollary 15.7 in [10]).

As an example of $A_{p}$-weight, the function $\omega(x)=|x|^{\alpha}, x \in \mathbb{R}^{n}$, is in $A_{p}$ if and only if $-n<\alpha<n(p-1)$ (see Corollary 4.4, Chapter IX in [14]).

Definition 2.2. Let $\omega$ be a weight, and let $\Omega \subset \mathbb{R}^{n}$ be open. For $1<p<\infty$ we define $L^{p}(\Omega, \omega)$ as the set of measurable functions $f$ on $\Omega$ such that

$$
\|f\|_{L^{p}(\Omega, \omega)}=\left(\int_{\Omega}|f(x)|^{p} \omega(x) d x\right)^{1 / p}<\infty .
$$


We denote $\left[L^{p}(\Omega, \omega)\right]^{n}=L^{p}(\Omega, \omega) \times \cdots \times L^{p}(\Omega, \omega)$.

If $\omega \in A_{p}, 1<p<\infty$, then $\omega^{-1 /(p-1)}$ is locally integrable and we have $L^{p}(\Omega, \omega) \subset L_{\text {loc }}^{1}(\Omega)$ for every open set $\Omega$ (see Remark 1.2.4 in [15]). It thus makes sense to talk about weak derivatives of functions in $L^{p}(\Omega, \omega)$.

Definition 2.3. Let $\Omega \subset \mathbb{R}^{n}$ be open, $k$ be a nonnegative integer and $\omega \in A_{p}(1<p<\infty)$. We define the weighted Sobolev space $W^{k, p}(\Omega, \omega)$ as the set of functions $u \in L^{p}(\Omega, \omega)$ with weak derivatives $D^{\alpha} u \in L^{p}(\Omega, \omega)$ for $1 \leq|\alpha| \leq k$. The norm of $u$ in $W^{k, p}(\Omega, \omega)$ is defined by

$$
\|u\|_{W^{k, p}(\Omega, \omega)}=\left(\int_{\Omega}|u(x)|^{p} \omega(x) d x+\sum_{1 \leq|\alpha| \leq k} \int_{\Omega}\left|D^{\alpha} u(x)\right|^{p} \omega(x) d x\right)^{1 / p} .
$$

We also define $W_{0}^{k, p}(\Omega, \omega)$ as the closure of $C_{0}^{\infty}(\Omega)$ with respect to the norm $\|\cdot\|_{W^{k, p}(\Omega, \omega)}$.

If $\omega \in A_{p}$, then $W^{k, p}(\Omega, \omega)$ is the closure of $C^{\infty}(\Omega)$ with respect to the norm (2.1) (see Theorem 2.1.4 in [15]). The spaces $W^{k, p}(\Omega, \omega)$ and $W_{0}^{k, p}(\Omega, \omega)$ are Banach spaces.

It is evident that the weight function $\omega$ which satisfies $0<c_{1} \leq \omega(x) \leq c_{2}$ for $x \in \Omega$ ( $c_{1}$ and $c_{2}$ positive constants), gives nothing new (the space $W_{0}^{k, p}(\Omega, \omega)$ is then identical with the classical Sobolev space $W_{0}^{k, p}(\Omega)$ ). Consequently, we shall be interested above in all such weight functions $\omega$ which either vanish somewhere in $\Omega \cup \partial \Omega$ or increase to infinity (or both).

The space $W_{0}^{1, p}(\Omega, \omega)$ is the closure of $C_{0}^{\infty}(\Omega)$ with respect to the norm (2.1). The dual space of $W_{0}^{1, p}(\Omega, \omega)$ is the space

$$
\begin{aligned}
& {\left[W_{0}^{1, p}(\Omega, \omega]^{*}=W^{-1, p^{\prime}}(\Omega, \omega)\right.} \\
= & \left\{T=f-\operatorname{div} G: G=\left(g_{1}, \cdots, g_{n}\right), \frac{f}{\omega}, \frac{g_{j}}{\omega} \in L^{p^{\prime}}(\Omega, \omega), j=1, \cdots, n\right\} .
\end{aligned}
$$

In this article we use the following results.

Lemma 2.1. Let $1<p<\infty$.

(a) There exists a constant $\alpha_{p}>0$ such that

$$
\left.|| x\right|^{p-2} x-|y|^{p-2} y\left|\leq \alpha_{p}\right| x-y \mid(|x|+|y|)^{p-2}, \quad \forall x, y \in \mathbb{R}^{n}
$$

(b) There exist two positive constants $\beta_{p}, \gamma_{p}$ such that for every $x, y \in \mathbb{R}^{n}$

$$
\beta_{p}(|x|+|y|)^{p-2}|x-y|^{2} \leq\left(|x|^{p-2} x-|y|^{p-2} y\right) \cdot(x-y) \leq \gamma_{p}(|x|+|y|)^{p-2}|x-y|^{2} .
$$

Proof. See [4], Proposition 17.2 and Proposition 17.3. 
Lemma 2.2. If $\omega \in A_{p}$, then

$$
\left(\frac{|E|}{|B|}\right)^{p} \leq C_{p, \omega} \frac{\mu(E)}{\mu(B)}
$$

whenever $B$ is a ball in $\mathbb{R}^{n}$ and $E$ is a measurable subset of $B$ (where $\mu(E)=\int_{E} \omega(x) d x$ ).

Proof. See Theorem 15.5 Strong doubling of $A_{p}$-weights in [10].

By Lemma 2.2, if $\omega \in A_{p}$, then $\mu(E)=0$ if and only if $|E|=0$; so there is no need to specify the measure when using the ubiquitous expression almost everywhere and almost every, both abbreviated a.e..

Theorem 2.1. Let $\omega \in A_{p}, 1<p<\infty$, and let $\Omega$ be a bounded open set in $\mathbb{R}^{n}$. If $u_{m} \rightarrow u$ in $L^{p}(\Omega, \omega)$ then there exist a subsequence $\left\{u_{m_{k}}\right\}$ and a function $\Phi \in L^{p}(\Omega, \omega)$ such that

(i) $u_{m_{k}}(x) \rightarrow u(x), m_{k} \rightarrow \infty$, a.e. on $\Omega$;

(ii) $\left|u_{m_{k}}(x)\right| \leq \Phi(x)$, a.e. on $\Omega$.

Proof. The proof of this theorem follows the lines of Theorem 2.8.1 in [7].

Theorem 2.2 (The weighted Sobolev inequality). Let $\Omega$ be an open bounded set in $\mathbb{R}^{n}$ and $\omega \in A_{p}, 1<p<\infty$. There exist positive constants $C_{\Omega}$ and $\delta$ such that for all $u \in W_{0}^{1, p}(\Omega, \omega)$ and all $\theta$ satisfying $1 \leq \theta \leq n /(n-1)+\delta$,

$$
\|u\|_{L^{p \theta}(\Omega, \omega)} \leq C_{\Omega}\|\nabla u\|_{L^{p}(\Omega, \omega)},
$$

where $C_{\Omega}$ depends only on $n, p$, the $A_{p}$-constant $C(p, \omega)$ of $\omega$ and the diameter of $\Omega$.

Proof. Its suffices to prove the inequality for functions $u \in C_{0}^{\infty}(\Omega)$ (see Theorem 1.3 in [6]). To extend the estimates (2.2) to arbitrary $u \in W_{0}^{1, p}(\Omega, \omega)$, we let $\left\{u_{m}\right\}$ be a sequence of $C_{0}^{\infty}(\Omega)$ functions tending to $u$ in $W_{0}^{1, p}(\Omega, \omega)$. Applying the estimates (2.2) to differences $u_{m_{1}}-u_{m_{2}}$, we see that $\left\{u_{m}\right\}$ will be a Cauchy sequence in $L^{p}(\Omega, \omega)$. Consequently the limit function $u$ will lie in the desired spaces and satisfy (2.2).

Definition 2.4. We denote by $X=W^{2, p}\left(\Omega, \omega_{1}\right) \cap W_{0}^{1, r}\left(\Omega, \omega_{2}\right)$ with the norm

$$
\|u\|_{X}=\||\nabla u|\|_{L^{r}\left(\Omega, \omega_{2}\right)}+\|\Delta u\|_{L^{p}\left(\Omega, \omega_{1}\right)} .
$$

Definition 2.5. We say that an element $u \in X$ is a (weak) solution of problem (P) if, for all $\varphi \in X$,

$$
\begin{aligned}
& \int_{\Omega}|\Delta u|^{p-2} \Delta u \Delta \varphi \omega_{1} d x+\int_{\Omega}|\Delta u|^{q-2} \Delta u \Delta \varphi v_{1} d x \\
& +\int_{\Omega}|\nabla u|^{r-2}\langle\nabla u, \nabla \varphi\rangle \omega_{2} d x+\int_{\Omega}|\nabla u|^{s-2}\langle\nabla u, \nabla \varphi\rangle v_{2} d x \\
= & \int_{\Omega} f(x) \varphi(x) d x+\int_{\Omega}\langle G, \nabla \varphi(x)\rangle d x .
\end{aligned}
$$


Remark 2.1. Since $2 \leq q, s<r<p<\infty$, if

$$
\frac{v_{1}}{\omega_{1}} \in L^{p /(p-q)}\left(\Omega, \omega_{1}\right) \text { and } \frac{v_{2}}{\omega_{2}} \in L^{r /(r-s)}\left(\Omega, \omega_{2}\right),
$$

there exist two constants $M_{1}>0, M_{2}>0$ such that

$$
\|u\|_{L^{q}\left(\Omega, v_{1}\right)} \leq M_{1}\|u\|_{L^{p}\left(\Omega, \omega_{1}\right)} \quad \text { and } \quad\|u\|_{L^{s}\left(\Omega, v_{2}\right)} \leq M_{2}\|u\|_{L^{r}\left(\Omega, \omega_{2}\right)},
$$

where

$$
\begin{aligned}
& M_{1}=\left[\int_{\Omega}\left(\frac{v_{1}}{\omega_{1}}\right)^{p /(p-q)} \omega_{1} d x\right]^{(p-q) / p q}=\left\|v_{1} / \omega_{1}\right\|_{L^{p /(p-q)}\left(\Omega, \omega_{1}\right)^{\prime}}^{1 / q} \\
& M_{2}=\left[\int_{\Omega}\left(\frac{v_{2}}{\omega_{2}}\right)^{r /(r-s)} \omega_{2} d x\right]^{(r-s) / r s}=\left\|v_{2} / \omega_{2}\right\|_{L^{r /(r-s)}\left(\Omega, \omega_{2}\right)}^{1 / s} .
\end{aligned}
$$

In fact, since $2 \leq q, s<r<p<\infty$, we have $\theta=p / q>1$ and $\theta^{\prime}=p /(p-q)$,

$$
\begin{aligned}
\|u\|_{L^{q}\left(\Omega, v_{1}\right)}^{q} & =\int_{\Omega}|u|^{q} v_{1} d x=\int_{\Omega}|u|^{q} \frac{v_{1}}{\omega_{1}} \omega_{1} d x \\
& \leq\left(\int_{\Omega}|u|^{q \theta} \omega_{1} d x\right)^{1 / \theta}\left(\int_{\Omega}\left(\frac{v_{1}}{\omega_{1}}\right)^{\theta^{\prime}} \omega_{1} d x\right)^{1 / \theta^{\prime}} \\
& =\left(\int_{\Omega}|u|^{p} \omega_{1} d x\right)^{q / p}\left(\int_{\Omega}\left(\frac{v_{1}}{\omega_{1}}\right)^{p /(p-q)} \omega_{1} d x\right)^{(p-q) / p} .
\end{aligned}
$$

Hence, $\|u\|_{L^{q}\left(\Omega, v_{1}\right)} \leq M_{1}\|u\|_{L^{p}\left(\Omega, \omega_{1}\right)}$.

Analogously, we obtain

$$
\|u\|_{L^{s}\left(\Omega, v_{2}\right)} \leq M_{2}\|u\|_{L^{r}\left(\Omega, \omega_{2}\right)} .
$$

Remark 2.2. In this paper, we will use many times the following Convergence Principle in Banach spaces: Let $X$ be a Banach space, $x \in X$ and a sequence $\left\{x_{n}\right\}$ in $X$. If every subsequence of $\left\{x_{n}\right\}$ has, in turn, a subsequence which converges strongly to $x$, then the original sequence converges strongly to $x$, i.e., $x_{n} \rightarrow x$ as $n \rightarrow \infty$ (see [16], Proposition 10.13).

\section{Proof of Theorem 1.1}

The basic idea is to reduce the problem $(\mathrm{P})$ to an operator equation $A u=T$ and apply the theorem below.

Theorem 3.1. Let $A: X \rightarrow X^{*}$ be a monotone, coercive and hemicontinuous operator on the real, separable, reflexive Banach space $X$. Then for each $T \in X^{*}$ the equation $A u=T$ has a solution $u \in X$. 
Proof. See Theorem 26.A in [17].

To prove the existence of solutions, we define $B, B_{1}, B_{2}, B_{3}, B_{4}: X \times X \rightarrow \mathbb{R}$ and $T:$ $X \rightarrow \mathbb{R}$ by

$$
\begin{aligned}
& B(u, \varphi)=B_{1}(u, \varphi)+B_{2}(u, \varphi)+B_{3}(u, \varphi)+B_{4}(u, \varphi), \\
& B_{1}(u, \varphi)=\int_{\Omega}|\Delta u|^{p-2} \Delta u \Delta \varphi \omega_{1} d x, \\
& B_{2}(u, \varphi)=\int_{\Omega}|\Delta u|^{q-2} \Delta u \Delta \varphi v_{1} d x, \\
& B_{3}(u, \varphi)=\int_{\Omega}|\nabla u|^{r-2}\langle\nabla u, \nabla \varphi\rangle \omega_{2} d x \\
& B_{4}(u, \varphi)=\int_{\Omega}|\nabla u|^{s-2}\langle\nabla u, \nabla \varphi\rangle v_{2} d x \\
& T(\varphi)=\int_{\Omega} f \varphi d x+\int_{\Omega}\langle G, \nabla \varphi\rangle d x .
\end{aligned}
$$

Then $u \in X$ is a (weak) solution to problem (P) if for all $\varphi \in X$

$$
B(u, \varphi)=B_{1}(u, \varphi)+B_{2}(u, \varphi)+B_{3}(u, \varphi)+B_{4}(u, \varphi)=T(\varphi) .
$$

Step 1. We define the operator $F_{1}: X \rightarrow L^{p^{\prime}}\left(\Omega, \omega_{1}\right)$ by

$$
\left(F_{1} u\right)(x)=|\Delta u(x)|^{p-2} \Delta u(x) .
$$

We now show that operator $F_{1}$ is bounded and continuous.

(i) We have

$$
\begin{aligned}
\left\|F_{1} u\right\|_{L^{p^{\prime}\left(\Omega, \omega_{1}\right)}}^{p^{\prime}} & =\int_{\Omega}\left|F_{1} u(x)\right|^{p^{\prime}} \omega_{1} d x=\left.\left.\int_{\Omega}|| \Delta u\right|^{p-2} \Delta u\right|^{p^{\prime}} \omega_{1} d x \\
& =\int_{\Omega}|\Delta u|^{p} \omega_{1} d x=\|\Delta u\|_{L^{p}\left(\Omega, \omega_{1}\right)}^{p} \leq\|u\|_{X}^{p} .
\end{aligned}
$$

Therefore, by (3.1) we obtain

$$
\left\|F_{1} u\right\|_{L^{p^{\prime}}\left(\Omega, \omega_{1}\right)} \leq\|u\|_{X}^{p-1}
$$

and hence the boundedness.

(ii) Let $u_{m} \rightarrow u$ in $X$ as $m \rightarrow \infty$. We need to show that $F_{1} u_{m} \rightarrow F_{1} u$ in $L^{p^{\prime}}\left(\Omega, \omega_{1}\right)$. If $u_{m} \rightarrow u$ in $X$, then $\left|\Delta u_{m}\right| \rightarrow|\Delta u|$ in $L^{p}\left(\Omega, \omega_{1}\right)$ and $\left|\nabla u_{m}\right| \rightarrow|\nabla u|$ in $L^{r}\left(\Omega, \omega_{2}\right)$. Using Theorem 2.1, there exist a subsequence $\left\{u_{m_{k}}\right\}$ and two functions $\Phi_{1} \in L^{p}\left(\Omega, \omega_{1}\right)$ and $\Phi_{2} \in L^{r}\left(\Omega, \omega_{2}\right)$ such that

$$
\begin{array}{ll}
\left|\nabla u_{m_{k}}(x)\right| \rightarrow|\nabla u(x)| & \text { a.e. in } \Omega, \\
\left|\nabla u_{m_{k}}(x)\right| \leq \Phi_{2}(x) & \text { a.e. in } \Omega, \\
\left|\Delta u_{m_{k}}(x)\right| \rightarrow|\Delta u(x)| & \text { a.e. in } \Omega, \\
\left|\Delta u_{m_{k}}(x)\right| \leq \Phi_{1}(x) & \text { a.e. in } \Omega .
\end{array}
$$


Now, since $p>2$, using (3.3c), (3.3d), $a=p / p^{\prime}=p-1$ and $a^{\prime}=(p-1) /(p-2)$, there exists a constant $\alpha_{p}>0$ (by Lemma 2.1(a)) such that

$$
\begin{aligned}
& \left\|F_{1} u_{m_{k}}-F_{1} u\right\|_{L^{p^{\prime}}\left(\Omega, \omega_{1}\right)}^{p^{\prime}}=\int_{\Omega}\left|F_{1} u_{m_{k}}-F_{1} u\right|^{p^{\prime}} \omega_{1} d x \\
= & \left.\int_{\Omega}|| \Delta u_{m_{k}}\right|^{p-2} \Delta u_{m_{k}}-\left.|\Delta u|^{p-2} \Delta u\right|^{p^{\prime}} \omega_{1} d x \\
\leq & \int_{\Omega}\left[\alpha_{p}\left|\Delta u_{m_{k}}-\Delta u\right|\left(\left|\Delta u_{m_{k}}\right|+|\Delta u|\right)^{p-2}\right]^{p^{\prime}} \omega_{1} d x \\
\leq & \alpha_{p}^{p^{\prime}} \int_{\Omega}\left|\Delta u_{m_{k}}-\Delta u\right|^{p^{\prime}}\left(2 \Phi_{1}\right)^{(p-2) p^{\prime}} \omega_{1} d x \\
= & 2^{(p-2) p^{\prime}} \alpha_{p}^{p^{\prime}} \int_{\Omega}\left|\Delta u_{m_{k}}-\Delta u\right|^{p^{\prime}} \Phi_{1}^{(p-2) p^{\prime}} \omega_{1} d x \\
\leq & 2^{(p-2) p^{\prime}} \alpha_{p}^{p^{\prime}}\left(\int_{\Omega}\left|\Delta u_{m_{k}}-\Delta u\right|^{p^{\prime} a} \omega_{1} d x\right)^{1 / a}\left(\int_{\Omega} \Phi_{1}^{(p-2) p^{\prime} a^{\prime}} \omega_{1} d x\right)^{1 / a^{\prime}} \\
= & 2^{(p-2) p^{\prime}} \alpha_{p}^{p^{\prime}}\left(\int_{\Omega}\left|\Delta u_{m_{k}}-\Delta u\right|^{p} \omega_{1} d x\right)^{p^{\prime} / p}\left(\int_{\Omega} \Phi_{1}^{p} \omega_{1} d x\right)^{(p-2) /(p-1)} \\
= & 2^{(p-2) p^{\prime}} \alpha_{p}^{p^{\prime}}\left\|\Delta u_{m_{k}}-\Delta u\right\|_{L^{p}\left(\Omega, \omega_{1}\right)}^{p^{\prime}}\left\|\Phi_{1}\right\|_{L^{p}\left(\Omega, \omega_{1}\right)}^{p^{\prime}(p-2)} \\
\leq & 2^{(p-2) p^{\prime}} \alpha_{p}^{p^{\prime}}\left\|u_{m_{k}}-u\right\|_{X}^{p^{\prime}}\left\|\Phi_{1}\right\|_{L^{p}\left(\Omega, \omega_{1}\right)}^{p^{\prime}(p-2)}
\end{aligned}
$$

Hence,

$$
\left\|F_{1} u_{m_{k}}-F_{1} u\right\|_{L^{p^{\prime}}(\Omega, \omega)} \leq 2^{p-2} \alpha_{p}\left\|u_{m_{k}}-u\right\|_{X}\left\|\Phi_{1}\right\|_{L^{p}\left(\Omega, \omega_{1}\right)}^{p-2} .
$$

Therefore (since $2<p<\infty$ ), we obtain $\left\|F_{1} u_{m_{k}}-F_{1} u\right\|_{L^{p^{\prime}}\left(\Omega, \omega_{1}\right)} \rightarrow 0$, that is,

$$
F_{1} u_{m_{k}} \rightarrow F_{1} u \text { in } L^{p^{\prime}}\left(\Omega, \omega_{1}\right) \text {. }
$$

By the Convergence Principle in Banach spaces (see Remark 2.2), we have

$$
F_{1} u_{m} \rightarrow F_{1} u \quad \text { in } L^{p^{\prime}}\left(\Omega, \omega_{1}\right) .
$$

Step 2. Define the operator $F_{2}: X \rightarrow L^{q^{\prime}}\left(\Omega, v_{1}\right),\left(F_{2} u\right)(x)=|\Delta u(x)|^{q-2} \Delta u(x)$. We also have that the operator $F_{2}$ is continuous and bounded. In fact:

(i) If $q>2$, we have by Remark 2.1

$$
\begin{aligned}
\left\|F_{2} u\right\|_{L^{q^{\prime}\left(\Omega, v_{1}\right)}}^{q^{\prime}} & =\left.\left.\int_{\Omega}|| \Delta u\right|^{q-2} \Delta u\right|^{q^{\prime}} v_{1} d x=\int_{\Omega}|\Delta u|^{q} v_{1} d x \\
& =\|\Delta u\|_{L^{q}\left(\Omega, v_{1}\right)}^{q} \leq M_{1}^{q}\|\Delta u\|_{L^{p}\left(\Omega, \omega_{1}\right)}^{q} \leq M_{1}^{q}\|u\|_{X}^{q} .
\end{aligned}
$$

Hence,

$$
\left\|F_{2} u\right\|_{L^{q^{\prime}}\left(\Omega, v_{1}\right)} \leq M_{1}^{q-1}\|u\|_{X}^{q-1}
$$


(ii) Now using (3.3c), (3.3d), Remark 2.1, $b=q / q^{\prime}=q-1$ and $b^{\prime}=(q-1) /(q-2)$ (if $q>2$ ), there exists a constant $\alpha_{q}>0$ (by Lemma 2.1(a)) such that

$$
\begin{aligned}
& \left\|F_{2} u_{m_{k}}-F_{2} u\right\|_{L^{q^{\prime}}\left(\Omega, v_{1}\right)}^{q^{\prime}}=\int_{\Omega}\left|F_{2} u_{m_{k}}-F_{2} u\right|^{q^{\prime}} v_{1} d x \\
= & \left.\int_{\Omega}|| \Delta u_{m_{k}}\right|^{q-2} \Delta u_{m_{k}}-\left.|\Delta u|^{q-2} \Delta u\right|^{q^{\prime}} v_{1} d x \\
\leq & \int_{\Omega}\left[\alpha_{q}\left|\Delta u_{m_{k}}-\Delta u\right|\left(\left|\Delta u_{m_{k}}\right|+|\Delta u|\right)^{(q-2)}\right]^{q^{\prime}} v_{1} d x \\
\leq & \alpha_{q}^{q^{\prime}} \int_{\Omega}\left|\Delta u_{m_{k}}-\Delta u\right|^{q^{\prime}}\left(2 \Phi_{1}\right)^{(q-2) q^{\prime}} v_{1} d x \\
\leq & 2^{(q-2) q^{\prime}} \alpha_{q}^{q^{\prime}}\left(\int_{\Omega}\left|\Delta u_{m_{k}}-\Delta u\right|^{q^{\prime} b} v_{1} d x\right)^{1 / b}\left(\int_{\Omega} \Phi_{1}^{(q-2) q^{\prime} b^{\prime}} v_{1} d x\right)^{1 / b^{\prime}} \\
= & \alpha_{q}^{q^{\prime}} 2^{(q-2) q^{\prime}}\left(\int_{\Omega}\left|\Delta u_{m_{k}}-\Delta u\right|^{q^{q}} v_{1} d x\right)^{q^{\prime} / q}\left(\int_{\Omega} \Phi_{1}^{q} v d x\right)^{(q-2) /(q-1)} \\
= & \alpha_{q}^{q^{\prime}} 2^{(q-2) q^{\prime}}\left\|\Delta u_{m_{k}}-\Delta u\right\|_{L^{q}\left(\Omega, v_{1}\right)}^{q^{\prime}}\left\|\Phi_{1}\right\|_{L^{q}\left(\Omega, v_{1}\right)}^{q^{\prime}((-2)} \\
\leq & \alpha_{q}^{q^{\prime}} 2^{(q-2) q^{\prime}} M_{1}^{q^{\prime}}\left\|\Delta u_{m_{k}}-\Delta u\right\|_{L^{p}\left(\Omega, \omega_{1}\right)}^{q^{\prime}} M_{1}^{q^{\prime}(q-2)}\left\|\Phi_{1}\right\|_{L^{p}\left(\Omega, \omega_{1}\right)}^{q^{\prime}(q-2)} \\
\leq & \alpha_{q}^{q^{\prime}} 2^{(q-2) q^{\prime}} M_{1}^{q}\left\|u_{m_{k}}-u\right\|_{X}^{q^{\prime}}\left\|\Phi_{1}\right\|_{L^{p}\left(\Omega, \omega_{1}\right)}^{q^{\prime}(q-2)} .
\end{aligned}
$$

Hence,

$$
\left\|F_{2} u_{m_{k}}-F_{2} u\right\|_{L^{q^{\prime}}\left(\Omega, v_{1}\right)} \leq 2^{q-2} \alpha_{q} M_{1}^{q-1}\left\|\Phi_{1}\right\|_{L^{p}\left(\Omega, \omega_{1}\right)}^{q-2}\left\|u_{m_{k}}-u\right\|_{X} .
$$

In the case $q=2$, we have $\left(F_{2} u\right)(x)=\Delta u(x)$. Hence,

$$
\begin{aligned}
& \left\|F_{2} u\right\|_{L^{2}\left(\Omega, v_{1}\right)}=\|\Delta u\|_{L^{2}\left(\Omega, v_{1}\right)} \leq M_{1}\|\Delta u\|_{L^{p}\left(\Omega, \omega_{1}\right)} \leq M_{1}\|u\|_{X^{\prime}} \\
& \left\|F_{2} u_{m_{k}}-F_{2} u\right\|_{L^{2}\left(\Omega, v_{1}\right)} \leq M_{1}\left\|\Delta u_{m_{k}}-\Delta u\right\|_{L^{p}\left(\Omega, \omega_{1}\right)} \leq M_{1}\left\|u_{m_{k}}-u\right\|_{X} .
\end{aligned}
$$

Therefore, for $2 \leq q<\infty$, we obtain $\left\|F_{2} u_{m_{k}}-F_{2} u\right\|_{L^{q^{\prime}}\left(\Omega, v_{1}\right)} \rightarrow 0$, that is,

$$
F_{2} u_{m_{k}} \rightarrow F_{2} u \text { in } L^{q^{\prime}}\left(\Omega, v_{1}\right)
$$

By the Convergence Principle in Banach spaces (see Remark 2.2), we have

$$
F_{2} u_{m} \rightarrow F_{2} u \text { in } L^{q^{\prime}}\left(\Omega, v_{1}\right) \text {. }
$$

Step 3. We define $F_{3}: X \rightarrow\left[L^{r^{\prime}}\left(\Omega, \omega_{2}\right)\right]^{n}$ by $\left(F_{3} u\right)(x)=|\nabla u(x)|^{r-2} \nabla u(x)$. We also have that the operator $F_{3}$ is continuous and bounded. In fact, we have 
(i) if $u \in X$, since $r>2$, we have

$$
\begin{aligned}
& \left\|F_{3} u\right\|_{L^{r^{\prime}\left(\Omega, \omega_{2}\right)}}^{r^{\prime}}=\int_{\Omega}\left|F_{3} u\right|^{r^{\prime}} \omega_{2} d x=\left.\left.\int_{\Omega}|| \nabla u\right|^{r-2} \nabla u\right|^{r^{\prime}} \omega_{2} d x \\
= & \int_{\Omega}|\nabla u|^{(r-1) r^{\prime}} \omega_{2} d x=\int_{\Omega}|\nabla u|^{r} \omega_{2} d x=\|\mid \nabla u\|_{L^{r}\left(\Omega, \omega_{2}\right)}^{r} \leq\|u\|_{X}^{r} .
\end{aligned}
$$

Hence,

$$
\left\|F_{3} u\right\|_{L^{r^{\prime}}\left(\Omega, \omega_{2}\right)} \leq\|u\|_{X}^{r-1}
$$

(ii) If $u_{m} \rightarrow u$ in $X$, then $\left|\nabla u_{m}\right| \rightarrow|\nabla u|$ in $L^{r}\left(\Omega, \omega_{2}\right)$. Using Theorem 2.1, there exists a subsequence $\left\{u_{m_{k}}\right\}$ and $\Phi_{2} \in L^{r}\left(\Omega, \omega_{2}\right)$ such that

$$
\begin{array}{ll}
\left|\nabla u_{m_{k}}\right| \rightarrow|\nabla u| & \text { a.e in } \Omega, \\
\left|\nabla u_{m_{k}}\right| \leq \Phi_{2} & \text { a.e in } \Omega .
\end{array}
$$

Hence, using (3.6a), (3.10), $\gamma=r / r^{\prime}=r-1$ and $\gamma^{\prime}=(r-1) /(r-2)$, there exists a constant $\alpha_{r}>0$ (by Lemma 2.1(a)) such that

$$
\begin{aligned}
& \left\|F_{3} u_{m_{k}}-F_{3} u\right\|_{L^{r^{\prime}}\left(\Omega, \omega_{2}\right)}^{r^{\prime}}=\int_{\Omega}\left|F_{3} u_{m_{k}}-F_{3} u\right|^{r^{\prime}} \omega_{2} d x \\
= & \left.\int_{\Omega}|| \nabla u_{m_{k}}\right|^{r-2} \nabla u_{m_{k}}-\left.|\nabla u|^{r-2} \nabla u\right|^{r^{\prime}} \omega_{2} d x \\
\leq & \int_{\Omega}\left[\alpha_{r}\left|\nabla u_{m_{k}}-\nabla u\right|\left(\left|\nabla u_{m_{k}}\right|+|\nabla u|\right)^{r-2}\right]^{r^{\prime}} \omega_{2} d x \\
\leq & \alpha_{r}^{r^{\prime}} \int_{\Omega}\left|\nabla u_{m_{k}}-\nabla u\right|^{r^{\prime}}\left(2 \Phi_{2}\right)^{(r-2) r^{\prime}} \omega_{2} d x \\
\leq & 2^{(r-2) r^{\prime}} \alpha_{r}^{r^{\prime}}\left(\int_{\Omega}\left|\nabla u_{m_{k}}-\nabla u\right|^{r^{\prime} \gamma} \omega_{2} d x\right)^{1 / \gamma}\left(\int_{\Omega} \Phi_{2}^{(r-2) r^{\prime} \gamma^{\prime}} \omega_{2} d x\right)^{1 / \gamma^{\prime}} \\
= & 2^{(r-2) r^{\prime}} \alpha_{r}^{r^{\prime}}\left\|\nabla u_{m_{k}}-\nabla u\right\|_{L^{r}\left(\Omega, \omega_{2}\right)}^{r^{\prime}}\left\|\Phi_{2}\right\|_{L^{r}\left(\Omega, \omega_{2}\right)}^{(r-2) r^{\prime}} \\
\leq & 2^{(r-2) r^{\prime}} \alpha_{r}^{r^{\prime}}\left\|u_{m_{k}}-u\right\|_{X}^{r^{\prime}}\left\|\Phi_{2}\right\|_{L^{r}\left(\Omega, \omega_{2}\right)}^{(r-2) r^{\prime}} .
\end{aligned}
$$

Hence,

$$
\left\|F_{3} u_{m_{k}}-F_{3} u\right\|_{L^{r^{\prime}}\left(\Omega, \omega_{2}\right)} \leq 2^{r-2} \alpha_{r}\left\|\Phi_{2}\right\|_{L^{r}\left(\Omega, \omega_{2}\right)}^{r-2}\left\|u_{m_{k}}-u\right\|_{X} .
$$

Therefore (since $2<r<\infty$ ), we obtain $\left\|F_{3} u_{m_{k}}-F_{3} u\right\|_{L^{\prime}\left(\Omega, \omega_{2}\right)} \rightarrow 0$ as $m_{k} \rightarrow \infty$, that is, $F_{3} u_{m_{k}} \rightarrow F_{u}$ in $L^{r^{\prime}}\left(\Omega, \omega_{2}\right)$. By the Convergence Principle in Banach spaces (see Remark 2.2), we obtain

$$
F_{3} u_{m} \rightarrow F_{3} u \text { in } L^{r^{\prime}}\left(\Omega, \omega_{2}\right) .
$$

Step 4. We define $F_{4}: X \rightarrow\left[L^{s^{\prime}}\left(\Omega, v_{2}\right)\right]^{n}$ by $\left(F_{4} u\right)(x)=|\nabla u(x)|^{s-2} \nabla u(x)$. We also have that the operator $F_{4}$ is continuous and bounded. In fact, we have 
(i) If $s>2$ and $u \in X$, by Remark 2.1, we obtain

$$
\begin{aligned}
& \left\|F_{4} u\right\|_{L^{s^{\prime}}\left(\Omega, v_{2}\right)}^{s^{\prime}}=\int_{\Omega}\left|F_{4} u\right|^{s^{\prime}} v_{2} d x=\left.\left.\int_{\Omega}|| \nabla u\right|^{s-2} \nabla u\right|^{s^{\prime}} v_{2} d x \\
= & \int_{\Omega}|\nabla u|^{(s-1) s^{\prime}} v_{2} d x=\int_{\Omega}|\nabla u|^{s} v_{2} d x=\|\mid \nabla u\|_{L^{s}\left(\Omega, v_{2}\right)}^{s} \\
\leq & M_{2}^{s}\||\nabla u|\|_{L^{r}\left(\Omega, \omega_{2}\right)}^{s} \leq M_{2}^{s}\|u\|_{X}^{s} .
\end{aligned}
$$

Hence,

$$
\left\|F_{4} u\right\|_{L^{s^{\prime}}\left(\Omega, v_{2}\right)} \leq M_{2}^{s-1}\|u\|_{X}^{s-1}
$$

(ii) If $u_{m} \rightarrow u$ in $X$, then $\left|\nabla u_{m}\right| \rightarrow|\nabla u|$ in $L^{r}\left(\Omega, \omega_{2}\right)$. Using Theorem 2.1, there exists a subsequence $\left\{u_{m_{k}}\right\}$ and $\Phi_{2} \in L^{r}\left(\Omega, \omega_{2}\right)$ such that

$$
\begin{array}{ll}
\left|\nabla u_{m_{k}}\right| \rightarrow|\nabla u| & \text { a.e in } \Omega, \\
\left|\nabla u_{m_{k}}\right| \leq \Phi_{2} & \text { a.e in } \Omega .
\end{array}
$$

Hence, using (3.8a), (3.8b), $\eta=s / s^{\prime}=s-1$ and $\eta^{\prime}=(s-1) /(s-2)$, there exists a constant $\alpha_{s}>0$ (by Lemma 2.1(a)) such that

$$
\begin{aligned}
& \left\|F_{4} u_{m_{k}}-F_{4} u\right\|_{L s^{\prime}\left(\Omega, v_{2}\right)}^{s^{\prime}}=\int_{\Omega}\left|F_{4} u_{m_{k}}-F_{4} u\right|^{s^{\prime}} v_{2} d x \\
= & \left.\int_{\Omega}|| \nabla u_{m_{k}}\right|^{s-2} \nabla u_{m_{k}}-\left.|\nabla u|^{s-2} \nabla u\right|^{s^{\prime}} v_{2} d x \\
\leq & \int_{\Omega}\left[\alpha_{s}\left|\nabla u_{m_{k}}-\nabla u\right|\left(\left|\nabla u_{m_{k}}\right|+|\nabla u|\right)^{s-2}\right]^{s^{\prime}} v_{2} d x \\
\leq & \alpha_{s}^{s^{\prime}} \int_{\Omega}\left|\nabla u_{m_{k}}-\nabla u\right|^{s^{\prime}}\left(2 \Phi_{2}\right)^{(s-2) s^{\prime}} v_{2} d x \\
\leq & 2^{(s-2) s^{\prime}} \alpha_{s}^{s^{\prime}}\left(\int_{\Omega}\left|\nabla u_{m_{k}}-\nabla u\right|^{s^{\prime} \eta} v_{2} d x\right)^{1 / \eta}\left(\int_{\Omega} \Phi_{2}^{(s-2) s^{\prime} \eta^{\prime}} v_{2} d x\right)^{1 / \eta^{\prime}} \\
= & 2^{(s-2) s^{\prime}} \alpha_{s}^{s^{\prime}}\left\|\left|\nabla u_{m_{k}}-\nabla u\right|\right\|_{L^{s}\left(\Omega, v_{2}\right)}^{s^{\prime}}\left\|\Phi_{2}\right\|_{L^{s}\left(\Omega, v_{2}\right)}^{(s-2) s^{\prime}} \\
\leq & 2^{(s-2) s^{\prime}} \alpha_{s}^{s^{\prime}} M_{2}^{s^{\prime}}\left\|\left|\nabla u_{m_{k}}-\nabla u\right|\right\|_{L^{r}\left(\Omega, \omega_{2}\right)}^{s^{\prime}} M_{2}^{s^{\prime}(s-2)}\left\|\Phi_{2}\right\|_{L^{r}\left(\Omega, \omega_{2}\right)}^{s^{\prime}(s-2)} \\
\leq & 2^{(s-2) s^{\prime}} \alpha_{s}^{s^{\prime}} M_{2}^{s^{\prime}(s-1)}\left\|u_{m_{k}}-u\right\|_{X}^{s^{\prime}}\left\|\Phi_{2}\right\|_{L^{r}\left(\Omega, \omega_{2}\right)}^{s^{\prime}(s-2)} .
\end{aligned}
$$

Hence,

$$
\left\|F_{4} u_{m_{k}}-F_{4} u\right\|_{L^{s^{\prime}}\left(\Omega, v_{2}\right)} \leq 2^{s-2} \alpha_{s} M_{2}^{s-1}\left\|\Phi_{2}\right\|_{L^{r}\left(\Omega, \omega_{2}\right)}^{s-2}\left\|u_{m_{k}}-u\right\|_{X} .
$$

In case $s=2$, we have $\left(F_{4}(u)\right)(x)=\nabla u$. Then

$$
\begin{aligned}
& \left\|F_{4} u\right\|_{L^{2}\left(\Omega, v_{2}\right)}=\||\nabla u|\|_{L^{2}\left(\Omega, v_{2}\right)} \leq M_{2}\||\nabla u|\|_{L^{2}\left(\Omega, \omega_{2}\right)} \leq M_{2}\|u\|_{X^{\prime}} \\
& \left\|F_{4} u_{m_{k}}-F_{4} u\right\|_{L^{2}\left(\Omega, v_{2}\right)} \leq M_{2}\left\|u_{m_{k}}-u\right\|_{X} .
\end{aligned}
$$


Therefore (for $2 \leq s<\infty$ ), we obtain that $\left\|F_{4} u_{m_{k}}-F_{4} u\right\|_{L^{s^{\prime}}\left(\Omega, v_{2}\right)} \rightarrow 0$ as $m_{k} \rightarrow \infty$, that is, $F_{4} u_{m_{k}} \rightarrow F_{4} u$ in $L^{s^{\prime}}\left(\Omega, v_{2}\right)$. By the Convergence Principle in Banach spaces (see Remark 2.2) we obtain

$$
F_{4} u_{m} \rightarrow F_{4} u \text { in } L^{s^{\prime}}\left(\Omega, v_{2}\right)
$$

Step 5. We also have, by (H3) and Theorem 2.2,

$$
\begin{aligned}
|T(\varphi)| & \leq \int_{\Omega}|f||\varphi| d x+\int_{\Omega}|G||\nabla \varphi| d x \\
& =\int_{\Omega} \frac{|f|}{\omega_{2}}|\varphi| \omega_{2} d x+\int_{\Omega} \frac{|G|}{\omega_{2}}|\nabla \varphi| \omega_{2} d x \\
& \leq\left\|f / \omega_{2}\right\|_{L^{r^{\prime}}\left(\Omega, \omega_{2}\right)}\|\varphi\|_{L^{r}\left(\Omega, \omega_{2}\right)}+\left\||G| / \omega_{2}\right\|_{L^{r^{\prime}\left(\Omega, \omega_{2}\right)}}\||\nabla \varphi|\| \|_{L^{r}\left(\Omega, \omega_{2}\right)} \\
& \leq C_{\Omega}\left\|f / \omega_{2}\right\|_{L^{r^{\prime}}\left(\Omega, \omega_{2}\right)}\||\nabla \varphi|\|_{L^{r}\left(\Omega, \omega_{2}\right)}+\left\||G| / \omega_{2}\right\|_{L^{r^{\prime}\left(\Omega, \omega_{2}\right)}}\||\nabla \varphi|\|_{L^{r}\left(\Omega, \omega_{2}\right)} \\
& \leq\left(C_{\Omega}\left\|f / \omega_{2}\right\|_{L^{r^{\prime}\left(\Omega, \omega_{2}\right)}}+\left\||G| / \omega_{2}\right\|_{L^{r^{\prime}}\left(\Omega, \omega_{2}\right)}\right)\|\varphi\|_{X^{\prime}}
\end{aligned}
$$

and $T \in\left[W_{0}^{1, r}\left(\Omega, \omega_{2}\right)\right]^{*} \subset X^{*}$ (i.e., $\left.T \in X^{*}\right)$. Moreover, we also have for all $u, \varphi \in X$

$$
|B(u, \varphi)| \leq\left|B_{1}(u, \varphi)\right|+\left|B_{2}(u, \varphi)\right|+\left|B_{3}(u, \varphi)\right|+\left|B_{4}(u, \varphi)\right| .
$$

Note that by Definition 2.4, we have

$$
\begin{aligned}
\left|B_{1}(u, \varphi)\right| & \leq \int_{\Omega}|\Delta u|^{p-1}|\Delta \varphi| \omega_{1} d x \\
& \leq\left(\int_{\Omega}|\Delta u|^{(p-1) p^{\prime}} \omega_{1} d x\right)^{1 / p^{\prime}}\left(\int_{\Omega}|\Delta \varphi|^{p} \omega_{1} d x\right)^{1 / p} \\
& =\|\Delta u\|_{L^{p}\left(\Omega, \omega_{1}\right)}^{p-1}\|\Delta \varphi\|_{L^{p}\left(\Omega, \omega_{1}\right)} \leq\|u\|_{X}^{p-1}\|\varphi\|_{X^{\prime}}
\end{aligned}
$$

by Remark 2.1

$$
\begin{aligned}
\left|B_{2}(u, \varphi)\right| & \leq \int_{\Omega}|\Delta u|^{q-1}|\Delta \varphi| v_{1} d x \\
& \leq\left(\int_{\Omega}|\Delta u|^{(q-1) q^{\prime}} v_{1} d x\right)^{1 / q^{\prime}}\left(\int_{\Omega}|\Delta \varphi|^{q} v_{1} d x\right)^{1 / q} \\
& =\|\Delta u\|_{L^{q}\left(\Omega, v_{1}\right)}^{q-1}\|\Delta \varphi\|_{L^{q}\left(\Omega, v_{1}\right)} \\
& \leq M_{1}^{q-1}\|\Delta u\|_{L^{p}\left(\Omega, \omega_{1}\right)}^{q-1} M_{1}\|\Delta \varphi\|_{L^{p}\left(\Omega, \omega_{1}\right)} \leq M_{1}^{q}\|u\|_{X}^{q-1}\|\varphi\|_{X^{\prime}}
\end{aligned}
$$


and by Definition 2.4,

$$
\begin{aligned}
\left|B_{3}(u, \varphi)\right| & \leq \int_{\Omega}|\nabla u|^{r-1}|\nabla \varphi| \omega_{2} d x \\
& \leq\left(\int_{\Omega}|\nabla u|^{(r-1) r^{\prime}} \omega_{2} d x\right)^{1 / r^{\prime}}\left(\int_{\Omega}|\nabla \varphi|^{r} \omega_{2} d x\right)^{1 / r} \\
& =\||\nabla u|\|_{L^{r}\left(\Omega, \omega_{2}\right)}^{r-1}\||\nabla \varphi|\|_{L^{r}\left(\Omega, \omega_{2}\right)} \leq\|u\|_{X}^{r-1}\|\varphi\|_{X} .
\end{aligned}
$$

Moreover, by Remark 2.1,

$$
\begin{aligned}
\mid B_{4}(u, \varphi) & \leq \int_{\Omega}|\nabla u|^{s-1}|\nabla \varphi| v_{2} d x \\
& \leq\left(\int_{\Omega}|\nabla u|^{(s-1) s^{\prime}} v_{2} d x\right)^{1 / s^{\prime}}\left(\int_{\Omega}|\nabla \varphi|^{s} v_{2} d x\right)^{1 / s} \\
& =\||\nabla u|\|_{L^{s}\left(\Omega, v_{2}\right)}^{s-1}\||\nabla \varphi|\|_{L^{s}\left(\Omega, v_{2}\right)} \\
& \leq M_{2}^{s-1}\||\nabla u|\|_{L^{r}\left(\Omega, \omega_{2}\right)}^{s-1} M_{2}\||\nabla \varphi|\|_{L^{r}\left(\Omega, \omega_{2}\right)} \leq M_{2}^{s}\|u\|_{x}^{s-1}\|\varphi\|_{X} .
\end{aligned}
$$

Consequently, we obtain in (3.10) that

$$
|B(u, \varphi)| \leq\left(\|u\|_{X}^{p-1}+M_{1}^{q}\|u\|_{X}^{q-1}+\|u\|_{X}^{r-1}+M_{2}^{s}\|u\|_{X}^{s-1}\right)\|\varphi\|_{X} .
$$

Since $B(u, \cdot)$ is linear, for each $u \in X$, there exists a linear and continuous functional on $X$ denoted by $A u$ such that $\langle A u, \varphi\rangle=B(u, \varphi)$, for all $u, \varphi \in X$ (where $\langle f, x\rangle$ denotes the value of the linear functional $f$ at the point $x$ ). Moreover,

$$
\|A u\|_{*} \leq\|u\|_{X}^{p-1}+M_{1}^{q}\|u\|_{X}^{q-1}+\|u\|_{X}^{r-1}+M_{2}^{s}\|u\|_{X}^{s-1},
$$

where $\|A u\|_{*}=\sup \left\{|\langle A u, \varphi\rangle|=|B(u, \varphi)|: \varphi \in X,\|\varphi\|_{X}=1\right\}$ is the norm of the operator $A u$.

Hence, we obtain the operator

$$
\begin{aligned}
A: X & \rightarrow X^{*}, \\
u & \mapsto A u .
\end{aligned}
$$

Consequently, problem $(\mathrm{P})$ is equivalent to the operator equation

$$
A u=T, \quad u \in X
$$


Step 6. If $u_{1}, u_{2} \in X$ we have by Lemma 2.1(b) and $2 \leq q, s<r<p<\infty$,

$$
\begin{aligned}
& \left\langle A u_{1}-A u_{2}, u_{1}-u_{2}\right\rangle=B\left(u_{1}, u_{1}-u_{2}\right)-B\left(u_{2}, u_{1}-u_{2}\right) \\
& =\int_{\Omega}\left(\left|\Delta u_{1}\right|^{p-1} \Delta u_{1}-\left|\Delta u_{2}\right|^{p-2} \Delta u_{2}\right) \Delta\left(u_{1}-u_{2}\right) \omega_{1} d x \\
& \quad+\int_{\Omega}\left(\left|\Delta u_{1}\right|^{q-1} \Delta u_{1}-\left|\Delta u_{2}\right|^{q-2} \Delta u_{2}\right) \Delta\left(u_{1}-u_{2}\right) v_{1} d x \\
& \quad+\int_{\Omega}\left\langle\left|\nabla u_{1}\right|^{r-1} \nabla u_{1}-\left|\nabla u_{2}\right|^{r-2} \nabla u_{2}, \nabla u_{1}-\nabla u_{2}\right\rangle \omega_{2} d x \\
& \quad+\int_{\Omega}\left\langle\left|\nabla u_{1}\right|^{s-1} \nabla u_{1}-\left|\nabla u_{2}\right|^{s-2} \nabla u_{2}, \nabla u_{1}-\nabla u_{2}\right\rangle v_{2} d x \\
& \geq \beta_{p} \int_{\Omega}\left(\left|\Delta u_{1}\right|+\left|\Delta u_{2}\right|\right)^{p-2}\left|\Delta u_{1}-\Delta u_{2}\right|^{2} \omega_{1} d x \\
& \quad+\beta_{q} \int_{\Omega}\left(\left|\Delta u_{1}\right|+\left|\Delta u_{2}\right|\right)^{q-2}\left|\Delta u_{1}-\Delta u_{2}\right|^{2} v_{1} d x \\
& \quad+\beta_{r} \int_{\Omega}\left(\left|\nabla u_{1}\right|+\left|\nabla u_{2}\right|\right)^{r-2}\left|\nabla u_{1}-\nabla u_{2}\right|^{2} \omega_{2} d x \\
& \quad+\beta_{s}\left(\left|\nabla u_{1}\right|+\left|\nabla u_{2}\right|\right)^{s-2}\left|\nabla u_{1}-\nabla u_{2}\right|^{2} v_{2} d x \\
& \quad \beta_{p} \int_{\Omega}\left(\left|\Delta u_{1}\right|+\left|\Delta u_{2}\right|\right)^{p-2}\left|\Delta u_{1}-\Delta u_{2}\right|^{2} \omega_{1} d x \\
& \quad+\beta_{r} \int_{\Omega}\left(\left|\nabla u_{1}\right|+\left|\nabla u_{2}\right|\right)^{r-2}\left|\nabla u_{1}-\nabla u_{2}\right|^{2} \omega_{2} d x \\
& \geq \beta_{p} \int_{\Omega}\left|\Delta u_{1}-\Delta u_{2}\right|^{p-2}\left|\Delta u_{1}-\Delta u_{2}\right|^{2} \omega_{1} d x \\
& \quad+\beta_{r} \int_{\Omega}\left|\nabla u_{1}-\nabla u_{2}\right|^{r-2}\left|\nabla u_{1}-\nabla u_{2}\right|^{2} \omega_{2} d x \\
& \int_{\Omega}\left|\Delta u_{1}-\Delta u_{2}\right|^{p} \omega_{1} d x+\beta_{r} \int_{\Omega}\left|\nabla u_{1}-\nabla u_{2}\right|^{r} \omega d x \geq 0 .
\end{aligned}
$$

Therefore, the operator $A$ is monotone. Moreover, we have

$$
\begin{aligned}
& \langle A u, u\rangle=B(u, u) \\
= & B_{1}(u, u)+B_{2}(u, u)+B_{3}(u, u)+B_{4}(u, u) \\
= & \int_{\Omega}|\Delta u|^{p} \omega_{1} d x+\int_{\Omega}|\Delta u|^{q} v_{1} d x+\int_{\Omega}|\nabla u|^{r} \omega_{2} d x+\int_{\Omega}|\nabla u|^{s} v_{2} d x \\
\geq & \int_{\Omega}|\Delta u|^{p} \omega_{1} d x+\int_{\Omega}|\nabla u|^{r} \omega_{2} d x \\
= & \|\Delta u\|_{L^{p}\left(\Omega, \omega_{2}\right)}^{p}+\||\nabla u|\|_{L^{r}\left(\Omega, \omega_{2}\right)}^{r} .
\end{aligned}
$$


Hence, since $2<r<p<\infty$, we have

$$
\frac{\langle A u, u\rangle}{\|u\|_{X}} \rightarrow+\infty \quad \text { as }\|u\|_{X} \rightarrow+\infty,
$$

that is, $A$ is coercive (using that $\lim _{t+a \rightarrow \infty} \frac{t^{p}+a^{r}}{t+a}=\infty$, with $t>0$ and $a>0$ ).

Step 7. We need to show that the operator $A$ is continuous. Let $u_{m} \rightarrow u$ in $X$ as $m \rightarrow \infty$. We have,

$$
\begin{aligned}
& \left|B_{1}\left(u_{m}, \varphi\right)-B_{1}(u, \varphi)\right| \\
\leq & \left.\int_{\Omega}|| \Delta u_{m}\right|^{p-2} \Delta u_{m}-\left|\Delta u_{m}\right|^{p-2} \Delta u|| \Delta \varphi \mid \omega_{1} d x \\
= & \int_{\Omega}\left|F_{1} u_{m}-F_{1} u \| \Delta \varphi\right| \omega_{1} d x \\
\leq & \left\|F_{1} u_{m}-F_{1} u\right\|_{L^{p^{\prime}\left(\Omega, \omega_{1}\right)}}\|\Delta \varphi\|_{L^{p}\left(\Omega, \omega_{1}\right)} \\
\leq & \left\|F_{1} u_{m}-F_{1} u\right\|_{L^{p^{\prime}\left(\Omega, \omega_{1}\right)}}\|\varphi\|_{X^{\prime}}
\end{aligned}
$$

and by Remark 2.1,

$$
\begin{aligned}
& \left|B_{2}\left(u_{m}, \varphi\right)-B_{2}(u, \varphi)\right| \\
& \leq\left.\int_{\Omega}|| \Delta u_{m}\right|^{q-2} \Delta u_{m}-|\Delta u|^{q-2} \Delta u|| \Delta \varphi \mid v_{1} d x \\
& =\int_{\Omega}\left|F_{2} u_{m}-F_{2} u\right||\Delta \varphi| v_{1} d x \\
& \leq\left\|F_{2} u_{m}-F_{2} u\right\|_{L^{q^{\prime}}\left(\Omega, v_{1}\right)}\|\Delta \varphi\|_{L^{q}\left(\Omega, v_{1}\right)} \\
& \leq M_{1}\left\|F_{2} u_{m}-F_{2} u\right\|_{L^{\prime}\left(\Omega, v_{1}\right)}\|\Delta \varphi\|_{L^{p}\left(\Omega, \omega_{1}\right)} \\
& \leq M_{1}\left\|F_{2} u_{m}-F_{2} u\right\|_{L^{q^{\prime}}\left(\Omega, \omega_{1}\right)}\|\varphi\|_{X^{\prime}}
\end{aligned}
$$

and

$$
\begin{aligned}
& \left|B_{3}\left(u_{m}, \varphi\right)-B_{3}(u, \varphi)\right| \\
\leq & \left.\int_{\Omega}|| \nabla u_{m}\right|^{r-2} \nabla u_{m}-|\nabla u|^{r-2} \nabla u|| \nabla \varphi \mid \omega_{2} d x \\
= & \int_{\Omega}\left|F_{3} u_{m}-F_{3} u \| \nabla \varphi\right| \omega_{2} d x \\
\leq & \left\|F_{3} u_{m}-F_{3} u\right\|_{L^{r^{\prime}}\left(\Omega, \omega_{2}\right)}\|\nabla \varphi\|_{L^{r}\left(\Omega, \omega_{2}\right)} \\
\leq & \left\|F_{3} u_{m}-F_{3} u\right\|_{L^{r^{\prime}}\left(\Omega, \omega_{2}\right)}\|\varphi\|_{X^{\prime}}
\end{aligned}
$$


and by Remark 2.1

$$
\begin{aligned}
& \left|B_{4}\left(u_{m}, \varphi\right)-B_{4}(u, \varphi)\right| \\
\leq & \left.\int_{\Omega}|| \nabla u_{m}\right|^{s-2} \nabla u_{m}-|\nabla u|^{s-2} \nabla u|| \nabla \varphi \mid v_{2} d x \\
= & \int_{\Omega}\left|F_{4} u_{m}-F_{4} u\right||\nabla \varphi| v_{2} d x \\
\leq & \left\|F_{4} u_{m}-F_{4} u\right\|_{L^{s^{\prime}}\left(\Omega, v_{2}\right)}\||\nabla \varphi|\|_{L^{s}\left(\Omega, v_{2}\right)} \\
\leq & M_{2}\left\|F_{4} u_{m}-F_{4} u\right\|_{L^{s^{\prime}}\left(\Omega, v_{2}\right)}\||\nabla \varphi|\|_{L^{r}\left(\Omega, \omega_{2}\right)} \\
\leq & M_{2}\left\|F_{4} u_{m}-F_{4} u\right\|_{L^{s^{\prime}}\left(\Omega, v_{2}\right)}\|\varphi\|_{X} .
\end{aligned}
$$

Hence,

$$
\begin{aligned}
& \quad\left|B\left(u_{m}, \varphi\right)-B(u, \varphi)\right| \\
& \leq\left|B_{1}\left(u_{m}, \varphi\right)-B_{1}(u, \varphi)\right|+\left|B_{2}\left(u_{m}, \varphi\right)-B_{2}(u, \varphi)\right| \\
& \quad+\left|B_{3}\left(u_{m}, \varphi\right)-B_{3}(u, \varphi)\right|+\left|B_{4}\left(u_{m}, \varphi\right)-B_{4}(u, \varphi)\right| \\
& \leq\left(\left\|F_{1} u_{m}-F_{1} u\right\|_{L^{p^{\prime}\left(\Omega, \omega_{1}\right)}}+M_{1}\left\|F_{2} u_{m}-F_{2} u\right\|_{L^{q^{\prime}}\left(\Omega, v_{1}\right)}\right. \\
& \left.\quad+\left\|F_{3} u_{m}-F_{3} u\right\|_{L^{r^{\prime}}\left(\Omega, \omega_{2}\right)}+M_{2}\left\|F_{4} u_{m}-F_{4} u\right\|_{L^{s^{\prime}}\left(\Omega, v_{2}\right)}\right)\|\varphi\|_{X} .
\end{aligned}
$$

Then we obtain

$$
\begin{aligned}
& \quad\left\|A u_{m}-A u\right\|_{*} \\
& \leq\left\|F_{1} u_{m}-F_{1} u\right\|_{L^{p^{\prime}}\left(\Omega, \omega_{1}\right)}+M_{1}\left\|F_{2} u_{m}-F_{2} u\right\|_{L^{q^{\prime}}\left(\Omega, v_{1}\right)} \\
& \quad+\left\|F_{3} u_{m}-F_{3} u\right\|_{L^{r^{\prime}}\left(\Omega, \omega_{2}\right)}+M_{2}\left\|F_{4} u_{m}-F_{4} u\right\|_{L^{s^{\prime}}\left(\Omega, v_{2}\right)} .
\end{aligned}
$$

Therefore, using (3.4), (3.5), (3.7) and (3.9), we have $\left\|A u_{m}-A u\right\|_{*} \rightarrow 0$ as $m \rightarrow+\infty$, that is, $A$ is continuous (and this implies that $A$ is hemicontinuous).

Therefore, by Theorem 3.1, the operator equation $A u=T$ has a solution $u \in X$ and it is a solution for problem $(\mathrm{P})$.

Step 8. Let us now prove the uniqueness of the solution. Suppose that $u_{1}, u_{2} \in X$ are two solutions of problem $(\mathrm{P})$. Then

$$
\begin{aligned}
& \int_{\Omega}\left|\Delta u_{i}\right|^{p-2} \Delta u_{i} \Delta \varphi \omega_{1} d x+\int_{\Omega}\left|\Delta u_{i}\right|^{q-2} \Delta u_{i} \Delta \varphi v_{1} d x \\
& \quad+\int_{\Omega}\left|\nabla u_{i}\right|^{r-2}\left\langle\nabla u_{i}, \nabla \varphi\right\rangle \omega_{2} d x+\int_{\Omega}\left|\nabla u_{i}\right|^{s-2}\left\langle\nabla u_{i}, \nabla \varphi\right\rangle v_{2} d x \\
& =\int_{\Omega} f \varphi d x+\int_{\Omega}\langle G, \nabla \varphi\rangle d x,
\end{aligned}
$$


for all $\varphi \in X$, and $i=1,2$. Hence,

$$
\begin{aligned}
& \int_{\Omega}\left(\left|\Delta u_{1}\right|^{p-2} \Delta u_{1}-\left|\Delta u_{2}\right|^{p-1} \Delta u_{2}\right) \Delta \varphi \omega_{1} d x \\
& \quad+\int_{\Omega}\left(\left|\Delta u_{1}\right|^{q-2} \Delta u_{1}-\left|\Delta u_{2}\right|^{q-1} \Delta u_{2}\right) \Delta \varphi v_{1} d x \\
& \quad+\int_{\Omega}\left\langle\left|\nabla u_{1}\right|^{r-2} \nabla u_{1}-\left|\nabla u_{2}\right|^{r-2} \nabla u_{2}, \nabla \varphi\right\rangle \omega_{2} d x \\
& \quad+\int_{\Omega}\left\langle\left|\nabla u_{1}\right|^{s-2} \nabla u_{1}-\left|\nabla u_{2}\right|^{s-2} \nabla u_{2}, \nabla \varphi\right\rangle v_{2} d x=0 .
\end{aligned}
$$

Therefore, we obtain for $\varphi=u_{1}-u_{2}$ and by Lemma 2.1(b) and $2<r<p<\infty$

$$
\begin{aligned}
0=\int_{\Omega} & \left(\left|\Delta u_{1}\right|^{p-2} \Delta u_{1}-\left|\Delta u_{2}\right|^{p-2} \Delta u_{2}\right) \Delta\left(u_{1}-u_{2}\right) \omega_{1} d x \\
& +\int_{\Omega}\left(\left|\Delta u_{1}\right|^{q-2} \Delta u_{1}-\left|\Delta u_{2}\right|^{q-2} \Delta u_{2}\right) \Delta\left(u_{1}-u_{2}\right) v_{1} d x \\
& +\int_{\Omega}\left\langle\left|\nabla u_{1}\right|^{r-2} \nabla u_{1}-\left|\nabla u_{2}\right|^{r-2} \nabla u_{2}, \nabla u_{1}-\nabla u_{2}\right\rangle \omega_{2} d x \\
& +\int_{\Omega}\left\langle\left|\nabla u_{1}\right|^{s-2} \nabla u_{1}-\left|\nabla u_{2}\right|^{s-2} \nabla u_{2}, \nabla u_{1}-\nabla u_{2}\right\rangle v_{2} d x \\
\geq \beta_{p} & \int_{\Omega}\left(\left|\Delta u_{1}\right|+\left|\Delta u_{2}\right|\right)^{p-2}\left|\Delta u_{1}-\Delta u_{2}\right|^{2} \omega_{1} d x \\
& +\beta_{q} \int_{\Omega}\left(\left|\Delta u_{1}\right|+\left|\Delta u_{2}\right|\right)^{q-2}\left|\Delta u_{1}-\Delta u_{2}\right|^{2} v_{1} d x \\
& +\beta_{r} \int_{\Omega}\left(\left|\nabla u_{1}\right|+\left|\nabla u_{2}\right|\right)^{r-2}\left|\nabla u_{1}-\nabla u_{2}\right|^{2} \omega_{2} d x \\
& +\beta_{s} \int_{\Omega}\left(\left|\nabla u_{1}\right|+\left|\nabla u_{2}\right|\right)^{s-2}\left|\nabla u_{1}-\nabla u_{2}\right|^{2} v_{2} d x \\
\geq \beta_{p} & \int_{\Omega}\left(\left|\Delta u_{1}\right|+\left|\Delta u_{2}\right|\right)^{p-2}\left|\Delta u_{1}-\Delta u_{2}\right|^{2} \omega_{1} d x \\
& +\beta_{r} \int_{\Omega}\left(\left|\nabla u_{1}\right|+\left|\nabla u_{2}\right|\right)^{r-2}\left|\nabla u_{1}-\nabla u_{2}\right|^{2} \omega_{2} d x \\
\geq \beta_{p} & \int_{\Omega}\left|\Delta u_{1}-\Delta u_{2}\right|^{p-2}\left|\Delta u_{1}-\Delta u_{2}\right|^{2} \omega_{1} d x \\
& +\beta_{r} \int_{\Omega}\left|\nabla u_{1}-\nabla u_{2}\right|^{r-2}\left|\nabla u_{1}-\nabla u_{2}\right|^{2} \omega_{2} d x \\
=\beta_{p} & \int_{\Omega}\left|\Delta u_{1}-\Delta u_{2}\right|^{p} \omega_{1} d x+\beta_{r} \int_{\Omega}\left|\nabla u_{1}-\nabla u_{2}\right|^{r} \omega_{2} d x
\end{aligned}
$$


Hence

$$
\left\|\nabla u_{1}-\nabla u_{2}\right\|_{L^{r}\left(\Omega, \omega_{2}\right)}=\left\|\Delta u_{1}-\Delta u_{2}\right\|_{L^{p}\left(\Omega, \omega_{1}\right)}=0 .
$$

Since $u_{1}, u_{2} \in X$, then $u_{1}=u_{2}$ a.e..

Step 9. Estimate for $\|u\|_{X}$. Since $u \in X$ is solution for problem (P), then for $\varphi=u$ in Definition 2.5, we have

$$
\begin{aligned}
& \int_{\Omega}|\Delta u|^{p} \omega_{1} d x+\int_{\Omega}|\Delta u|^{q} v_{1} d x+\int_{\Omega}|\nabla u|^{r} \omega_{2} d x+\int_{\Omega}|\nabla u|^{s} v_{2} d x \\
= & \int_{\Omega} f u d x+\int_{\Omega}\langle G, \nabla u\rangle d x .
\end{aligned}
$$

Hence,

$$
\begin{aligned}
& \int_{\Omega}|\Delta u|^{p} \omega_{1} d x+\int_{\Omega}|\nabla u|^{r} \omega_{2} d x \\
\leq & \int_{\Omega}|\Delta u|^{p} \omega_{1} d x+\int_{\Omega}|\Delta u|^{q} v_{1} d x+\int_{\Omega}|\nabla u|^{r} \omega_{2} d x+\int_{\Omega}|\nabla u|^{s} v_{2} d x \\
= & \int_{\Omega} f u d x+\int_{\Omega}\langle G, \nabla u\rangle d x \\
\leq & C_{\Omega}\left\|f / \omega_{2}\right\|_{L^{r^{\prime}\left(\Omega, \omega_{2}\right)}}\||\nabla u|\|_{L^{r}\left(\Omega, \omega_{2}\right)}+\left\||G| / \omega_{2}\right\|_{L^{r^{\prime}\left(\Omega, \omega_{2}\right)}}\||\nabla u|\|_{L^{r}\left(\Omega, \omega_{2}\right)} \\
\leq & \left(C_{\Omega}\left\|f / \omega_{2}\right\|_{L^{r^{\prime}\left(\Omega, \omega_{2}\right)}}+\left\||G| / \omega_{2}\right\|_{L^{r^{\prime}\left(\Omega, \omega_{2}\right)}}\right)\|u\|_{X} \\
= & M\|u\|_{X^{\prime}}
\end{aligned}
$$

where

$$
M=C_{\Omega}\left\|f / \omega_{2}\right\|_{L^{r^{\prime}}\left(\Omega, \omega_{2}\right)}+\left\||G| / \omega_{2}\right\|_{L^{r^{\prime}}\left(\Omega, \omega_{2}\right)} .
$$

With that we obtain

$$
\int_{\Omega}|\Delta u|^{p} \omega_{1} d x \leq M\|u\|_{X} \quad \text { and } \quad \int_{\Omega}|\nabla u|^{r} \omega_{2} d x \leq M\|u\|_{X} .
$$

Therefore, by Young's inequality, we obtain

$$
\begin{aligned}
\|u\|_{X} & =\|\Delta u\|_{L^{p}\left(\Omega, \omega_{1}\right)}+\||\nabla u|\|_{L^{r}\left(\Omega, \omega_{2}\right)} \\
& \leq M^{1 / p}\|u\|_{X}^{1 / p}+M^{1 / r}\|u\|_{X}^{1 / r} \\
& \leq \frac{M^{p^{\prime} / p}}{p^{\prime}}+\frac{\|u\|_{X}}{p}+\frac{M^{r^{\prime} / r}}{r^{\prime}}+\frac{\|u\|_{X}}{r} \\
& =\frac{M^{p^{\prime} / p}}{p^{\prime}}+\frac{M^{r^{\prime} / r}}{r^{\prime}}+\left(\frac{1}{p}+\frac{1}{r}\right)\|u\|_{X} .
\end{aligned}
$$

Hence, since

$$
0<\frac{1}{p}+\frac{1}{r}<1
$$


we obtain

$$
\|u\|_{X} \leq C_{p, r}\left(\frac{M^{p^{\prime}-1}}{p^{\prime}}+\frac{M^{r^{\prime}-1}}{r^{\prime}}\right),
$$

where $C_{p, r}=p r /(p r-p-r)$. This completes the proof of Theorem 1.1.

Example 3.1. Let $\Omega=\left\{(x, y) \in \mathbb{R}^{2}: x^{2}+y^{2}<1\right\}$. Consider $q=s=2, r=3$ and $p=4$. Let $\omega_{1}(x, y)=\left(x^{2}+y^{2}\right)^{5 / 2}, \omega_{2}(x, y)=\left(x^{2}+y^{2}\right)^{-1 / 2}, v_{1}(x, y)=\left(x^{2}+y^{2}\right)^{2}$ and $v_{2}(x, y)=\left(x^{2}+y^{2}\right)^{-1 / 3}$ (we have $\omega_{1} \in A_{4}$ and $\omega_{2} \in A_{3}$ ). Let us consider the partial differential operator

$$
L u(x, y)=\Delta\left[\omega_{1}(x, y)|\Delta u|^{2} \Delta u+v_{1}(x, y) \Delta u\right]-\operatorname{div}\left[\omega_{2}(x, y)|\nabla u| \nabla u+v_{2}(x, y) \nabla u\right] .
$$

Therefore, by Theorem 1.1, the problem

$$
(P) \begin{cases}L u(x)=\frac{\cos (x y)}{\sqrt{x^{2}+y^{2}}}-\frac{\partial}{\partial x}\left(\frac{\sin (x y)}{\sqrt{x^{2}+y^{2}}}\right)-\frac{\partial}{\partial y}\left(\frac{\sin (x y)}{\sqrt{x^{2}+y^{2}}}\right) & \text { in } \Omega, \\ u(x)=\Delta u(x)=0 & \text { on } \partial \Omega,\end{cases}
$$

has a unique solution $u \in X=W^{2,4}\left(\Omega, \omega_{1}\right) \cap W_{0}^{1,3}\left(\Omega, \omega_{2}\right)$.

\section{Acknowledgements}

The author would like to thank the referee for his/her useful suggestions and comments which have improved the presentation of the paper.

\section{References}

[1] A. C. Cavalheiro, Existence and uniqueness of solutions for some degenerate nonlinear Dirichlet problems, J. Appl. Anal., 19 (2013), 41-54.

[2] A.C. Cavalheiro, Existence results for Dirichlet problems with degenerated $p$-Laplacian and p-Biharmonic operators, Appl. Math. E-Notes, 13 (2013), 234-242.

[3] A. C. Cavalheiro, Topics on Degenerate Elliptic Equations, Lambert Academic Publishing, Germany, (2018).

[4] M. Chipot, Elliptic Equations: An Introductory Course, Birkhäuser, Berlin,(2009).

[5] P. Drábek, A. Kufner and F. Nicolosi, Quasilinear Elliptic Equations with Degenerations and Singularities, Walter de Gruyter, Berlin, (1997).

[6] E. Fabes, C. Kening and R. Serapion, The local regularity of solutions of degenerate elliptic equations, Commun. PDEs, 7 (1982), 77-116.

[7] S. Fučik, O. John and A. Kufner, Function Spaces, Noordhoff International Publ., Leyden, (1977).

[8] J. Garcia-Cuerva and J. L. Rubio de Francia, Weighted Norm Inequalities and Related Topics, North-Holland Mathematics Studies, 116 (1985). 
[9] D. Gilbarg and N. S. Trudinger, Elliptic Partial Equations of Second Order, 2nd Ed., Springer, New York, (1983).

[10] J. Heinonen, T. Kilpeläinen and O. Martio, Nonlinear Potential Theory of Degenerate Elliptic Equations, Oxford Math. Monographs, Clarendon Press, (1993).

[11] A. Kufner, Weighted Sobolev Spaces, John Wiley \& Sons, (1985).

[12] B. Muckenhoupt, Weighted norm inequalities for the Hardy maximal function, Trans. Amer. Math. Soc., 165 (1972), 207-226.

[13] M. Talbi and N. Tsouli, On the spectrum of the weighted $p$-Biharmonic operator with weight, Mediterr. J. Math., 4 (2007), 73-86.

[14] A. Torchinsky, Real-Variable Methods in Harmonic Analysis, Academic Press, San Diego, (1986).

[15] B. O. Turesson, Nonlinear Potential Theory and Weighted Sobolev Spaces, Lecture Notes in Math., vol. 1736, Springer-Verlag, (2000).

[16] E. Zeidler, Nonlinear Functional Analysis and Its Applications, vol. I, Springer-Verlag, (1990).

[17] E. Zeidler, Nonlinear Functional Analysis and Its Applications, vol. II/B, Springer-Verlag, (1990). 